УДК 519.624

В. С. Дейцека, I. В. Сергієıко, В. В. Скопецький

(Іи-г кіберпетики НАН Украйни, Киї)

\title{
ЗАДАЧІ НА ВЛАСНІ ЗНАЧЕННЯ З РОЗРИВНИМИ ВЛАСНИМИ ФУНКЩІЯМИ ТА ÏХ ЧИСЕЛЬНІ РОЗВ'ЯЗКИ
}

We consider new eigenvalue problems with discontinuous eigen-functions. We construct computational algorithms which are not worse in precision than similar ones known for problems with smooth eigenfunctions.

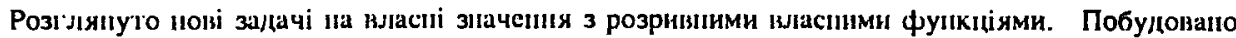

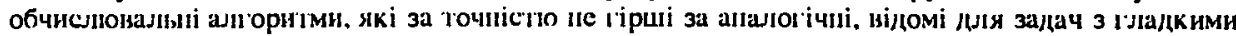

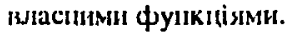

Проектування об'єктів довільного призначення, що вміщують тонкі прошарки матеріалів, які суттєво відрізцяюоться своїмн фізичними характеристиками від відповідних характеристик основного середовища, вимагає знаходження розрнвних розв'язків рівняньь з частинниим похідними [1-3].

В даній статті розглянуто задачі на власні значення з розривннми власними функціями елііттнчного рівняння другого порядку, запнсаного в декартовій та циліндрнчній снстемах коордннат. Для днскретизації цих задач запрогоновано обчислювалын схеми методу скінченних елементів (МСЕ), що базуються на використанні класів кусково-розрнвних функцій [4]. Асимптотична точність запропонованих обчислювалынх схем пе гірша за апалогічні, відомі для задач з гладкнми власними функціямн [5].

Розглянемо задачі на власні заяення з розрнвиими власними функціями.

Задача І. Нехай в обмежепій області $\Omega$, що складається $з$ двох областей

$$
\Omega_{1}, \Omega_{2}, \Omega=\bigcup_{l=1}^{2} \Omega_{l}, \quad \Omega_{1} \cap \Omega_{2}=\varnothing, \quad \bar{\Omega}_{1} \cap \bar{\Omega}_{2}=\gamma, \quad \Gamma=\partial \Omega_{1} \cup \partial \Omega_{2} \backslash \gamma
$$

( $\bar{\Omega}_{l}$-багатокутникн з кусково-лінійнимн без самоперетинів межами $\partial \Omega_{l}, l=1$, 2), визначено рівняння

$$
-\sum_{i . j=1}^{2} \frac{\partial}{\partial x_{i}}\left(k_{i j}(x) \frac{\partial u}{\partial x_{j}}\right)+q u=\lambda \rho u, \quad x \in \Omega .
$$

3. крайовнмин умовамін

$$
u=0, \quad x \in \Gamma .
$$

Тут $x=\left(x_{1}, x_{2}\right), k_{i j}=k_{j i} \in C\left(\bar{\Omega}_{l}\right) \cap C^{1}\left(\Omega_{l}\right),\left|k_{i j}^{\prime}\right|<\infty ; \rho, q \in C\left(\Omega_{l}\right), 0 \leq q \leq$ $\leq q_{1}<\infty, 0<\rho_{0} \leq \rho \leq \rho_{1}<\infty, l=1,2, i, j=1,2$,

$$
\sum_{i, j=1}^{2} k_{i j} \xi_{i} \xi_{j} \geq \alpha_{1} \sum_{i=1}^{2} \xi_{i}^{2} \quad \forall \xi_{i}, \xi_{j} \in R, \quad \alpha_{1}>0 .
$$

Умови спряження „неідеального" контакту мають вигляд

$$
\begin{gathered}
{\left[\sum_{i, j=1}^{2} k_{i j} \frac{\partial u}{\partial x_{j}} \cos \left(n, x_{i}\right)\right]=0, \quad x \in \gamma,} \\
\left\{\sum_{i, j=1}^{2} k_{i j} \frac{\partial u}{\partial x_{j}} \cos \left(n, x_{i}\right)\right\}^{ \pm}=R[u], \quad x \in \gamma,
\end{gathered}
$$

де $\{\varphi\}^{ \pm}=\varphi^{ \pm} ;[\varphi]=\varphi^{+}-\varphi^{-}$при $x \in \gamma$. Тут $\varphi^{-}=\varphi(x)$ при $x \in \partial \Omega_{1} \cap \gamma, \varphi^{+}=$ $=\varphi(x)$ при $x \in \partial \Omega_{2} \cap \gamma ; n$ - нормаль до $\gamma$, що напрямлена в область $\Omega_{2}$.

Задача II. Нехай у згаданій вище обмеженій області $\Omega$, що складається 3 
двох областей $\Omega_{1}, \Omega_{2}$, визначено рівняння

$$
-\frac{\partial}{\partial x_{1}}\left(x_{1} k_{1}(x) \frac{\partial u}{\partial x_{1}}\right)-\frac{\partial}{\partial x_{2}}\left(x_{1} k_{2}(x) \frac{\partial u}{\partial x_{2}}\right)=\lambda x_{1} \rho u, \quad x \in \Omega,
$$

3 крайовими умовами

$$
u=0, \quad x \in \Gamma .
$$

Умови спряження „неідеального" контакту на відрізку $\gamma$ дотнку областей $\Omega_{1}$, $\Omega_{2}$ мають вигляд

$$
\begin{gathered}
{\left[\sum_{i=1}^{2} k_{i} \frac{\partial u}{\partial x_{i}} \cos \left(n, x_{i}\right)\right]=0, \quad x \in \gamma,} \\
\left\{\sum_{i=1}^{2} k_{i} \frac{\partial u}{\partial x_{i}} \cos \left(n, x_{i}\right)\right\}^{ \pm}=R[u], \quad x \in \gamma,
\end{gathered}
$$

де $k_{i} \in C\left(\bar{\Omega}_{l}\right) \cap C^{1}\left(\Omega_{l}\right) ; l=1,2 ; 0<\dot{k}^{0} \leq k_{i} \leq k^{1}<\infty$.

Зауважения. При розгляді задачі (5)-(8) вважаємо, що області $\bar{\Omega}_{1}, \bar{\Omega}_{2} \epsilon$ такими, де виконується умова $\forall x \in \bar{\Omega} \Rightarrow x_{1} \geq \alpha>0$. В задачі (1)-(4) виконання цієі умови не вимагається.

Для задач I, II ((1)-(4); (5)-(8)) введемо в розгляд лінійні множини $M^{v}(v=$ $=\mathrm{I}, \Pi$ ) функцій $v(x)$, які неперервні; неперервно диференціновні на $\bar{\Omega}_{l}, l=1$, 2 , мають неперервні обмежені другі частинні похідні на $\Omega_{l}$ і задовольняють відповідно умови (2)-(4) і (6)-(8). Множини $M^{\nu}-$ щільні в $L_{2}(\Omega)$ [6]. Тоді краң̆ові задачі I, II полягають в тому, що необхідно знайти чнсла $\lambda^{v}$ і відмінні від нульових функції $\iota^{v}(x) \in M^{v}$, які задовольняють диференціальне рівняння (1) (задачі I), або рівняння (5) (задачі II).

Легко бачити, що власні функції $u^{v}(x) \in M^{v}$ задач I, II допускають розрив першого роду на відрізку $\gamma$ дотику областей $\Omega_{1}, \Omega_{2}$. Диференціальні оператори $L^{v}$ (при $v=I$ визначаються лівою частиною рівняння (1), а при $v=I I-$ лівою частиною рівняння (5)) - симетричні і додатно визначені на $M^{\mathrm{v}} \subset L_{2}(\Omega)$ [4]. Tyr

$$
\begin{aligned}
& \left(L^{\mathrm{I}} u, v\right)=\iint_{\Omega}\left\{\sum_{i, j=1}^{2} k_{i j} \frac{\partial u}{\partial x_{j}} \frac{\partial v}{\partial x_{i}}+q u v\right\} d \Omega+R \int_{\gamma}[u][v] d \gamma= \\
& =\left(u, L^{1} v\right)=\alpha^{1}(u, v) \quad \forall u, v \in M^{l}, \\
& \left(L^{\prime} u, u\right)=\alpha^{1}(u, u) \geq \alpha_{1} \iint_{\Omega} \sum_{i=1}^{2}\left(\frac{\partial u}{\partial x_{i}}\right)^{2} d \Omega \geq \alpha_{1} \mu_{1} \iint_{\Omega} u^{2} d \Omega ; \\
& \left(L^{\mathrm{II}} u, v\right)=\iint_{\Omega} x_{1} \sum_{i=1}^{2} k_{i} \frac{\partial u}{\partial x_{i}} \frac{\partial v}{\partial x_{i}} d \Omega+R \int_{\gamma} x_{1}[u][v] d \gamma= \\
& =\left(u, L^{\prime \prime} v\right)=\alpha^{\prime \prime}(u, v) \quad \forall u, v \in \dot{M}^{\prime \prime} \text {, } \\
& \left(L^{\mathrm{Il}} u, u\right)=\alpha^{\mathrm{II}}(u, u) \geq \alpha k^{0} \iint_{\Omega} \sum_{i=1}^{2}\left(\frac{\partial u}{\partial x_{i}}\right)^{2} d \Omega \geq \alpha k^{0} \mu_{1} \iint_{\Omega} u^{2} d \Omega,
\end{aligned}
$$

де $\mu_{1}>0$ - стала в узагальненій нерівності Фрідріхса $[4,6]$ : 


$$
\iint_{\Omega} \sum_{i=1}^{2}\left(\frac{\partial u}{\partial x_{i}}\right)^{2} d \Omega \geq \mu_{1} \iint_{\Omega} u^{2} d \Omega \quad \forall u \in M^{v}, \quad \mathrm{~V}=\mathrm{I}, \mathrm{II} .
$$

На $M^{v}$ введемо в розгляц епергетичинці скалярниї добуток $\langle\cdot, \cdot\rangle^{v}$ і енергетічну порму $\|\cdot\|_{L^{v}}$ гакім чнном:

$$
\begin{gathered}
\langle u, v\rangle^{v}=\alpha^{v}(u, v) \quad \forall u, v \in M^{v}, \\
\|u\|_{L^{v}}=\left\{\alpha^{v}(u, u)\right\}^{1 / 2} \quad \forall u \in M^{v}, \quad v=\text { I, II } .
\end{gathered}
$$

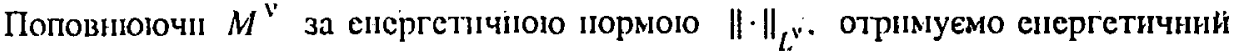
Ipocrip $H_{\mathrm{v}} \subset L_{2}(\Omega)[6]$.

Дотримуіочись, [5. 6], лля кожної з задач I, II отримуємо по дві еквівалентні узагальені задачі па власиі пачения з власними фупкціями, цо допускапоть розрив на $\gamma$.

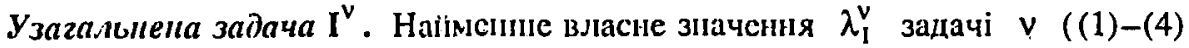
чІІ (5)-(8)) внзипчаеться як паймспнтс згачепня фупкціонала Рслєя

$$
R^{v}(v)=\frac{\|v\|_{! v}^{2}}{b^{v}(v, v)} \quad \forall v \in H_{v}
$$

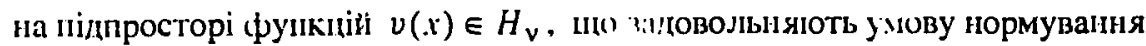

$$
b^{v}(v, v)=1, \quad v \in H_{v}, \quad v=\text { I. II, }
$$

цLe

$$
\begin{gathered}
b^{\mathrm{I}}(v, v)=\iint_{\Omega} \rho v^{2} d \Omega, \quad b^{\mathrm{II}}(v, v)=\iint_{\Omega} x_{1} \rho v^{2} d \Omega, \\
\lambda_{1}^{v}=\frac{\left\|v_{1}^{v}\right\|_{L^{v}}}{b^{v}\left(v_{1}^{v}, v_{1}^{v}\right)}, \quad v_{1}^{v}(x) \in H_{v} .
\end{gathered}
$$

Всі іпші власпі значення $\lambda_{m}^{v}, m>1$, задачі $v$ в порядку іх зростання визиачаються як діисні числа, які є наймепшими зачсниямн функціонала Релєя $R^{v}(v)(11)$ на підпросторах $H_{m}^{v} \subset H_{\mathrm{v}}$ функцій $v(x)$, що задовольняготь умови

$$
b^{v}(v, v)=1, \quad b^{v}\left(v, v_{l}^{v}\right)=0, \quad l=\overline{1, m-1}, \quad v_{l}^{v} \in H_{v},
$$

це $v_{l}^{v}(x)$ - власна функція задачі $v$, ццо відповідає власному значенноо $\lambda_{l}^{v}$.

Узагалынена задача II $^{v}$ полягае в тому, що пеобхідно знайти $\lambda^{v}$ і відмінні від нульовгх функції $u^{v}(x) \neq 0, u^{v} \in H_{v}$, такі, що для будь-якого $v(x) \in H_{\mathrm{v}}$ внконуеться рівність

$$
\left\langle u^{v}, v\right\rangle^{v}=\lambda^{v} b^{v}\left(u^{v}, v\right), \quad u^{v} \in H_{v} \quad \forall v \in H_{v}, \quad v=\mathrm{I}, \mathrm{II} .
$$

Справедлива така лема.

Лема 1. Власні функиіi $v_{\| t}^{v}(x), v_{m}^{v}(x) \in H_{v}$ задач I, II, що допускають розрия на $\gamma$, ортогональні між собою в сенсі скалярних добутків $\langle\cdot, \cdot\rangle^{v}$, $b^{v}(\cdot, \cdot) n p u \lambda_{n}^{v} \neq \lambda_{m}^{v}$.

Доведения. Враховуіочи співвідношення (12), маємо 


$$
\left\langle v_{n}^{v}, v_{m}^{v}\right\rangle^{v}=\lambda_{n}^{v} b^{v}\left(v_{n}^{v}, v_{m}^{v}\right), \quad\left\langle v_{m}^{v}, v_{n}^{v}\right\rangle^{v}=\lambda_{m}^{v} b^{v}\left(v_{m}^{v}, v_{n}^{v}\right)
$$

Oтже,

$$
0=\left(\lambda_{n}^{v}-\lambda_{m}^{v}\right) b^{v}\left(v_{n}^{v}, v_{m}^{v}\right) \Rightarrow b^{v}\left(v_{n}^{v}, v_{m}^{v}\right)=0
$$

Kрім того,

$$
\left\langle v_{n}^{v}, v_{m}^{v}\right\rangle^{v}=\lambda_{n}^{v} b^{v}\left(v_{n}^{v}, v_{m}^{v}\right)=0
$$

Лему доведено.

Твердження. Якцо власна функція $u_{n}^{\mathrm{v}}(x) \in H^{\mathrm{v}}$, цо допускає розрив на $\gamma$, відповідної узагальненої задаці належить множині $C^{\prime}\left(\bar{\Omega}_{l}\right) \cap C^{2}\left(\Omega_{l}\right) . l=1,2$, то вона задовольняе умьви спряхенкя неідеального контакту (умови (3), (4) в задачі I, чи (7), (8) - в задачі II).

Доведення. Нехай $u_{n}^{\vee}(x)$ - власна функція, що допускає розрив на $\gamma$, яка відповідає власному значенню $\lambda_{n}^{v}$ задачі $v$, а функція $v(x) \in \stackrel{\circ}{C}^{\infty}\left(\Omega_{l}\right)$ і $v(x) \equiv 0$ при $x \in \bar{\Omega}_{2}$. Тоді з (12) отримуємо

$$
\iint_{\Omega_{1}}\left\{\Psi^{v} \sum_{i, j=1}^{2} k_{i j}^{v} \frac{\partial u_{n}^{v}}{\partial x_{j}} \frac{\partial v}{\partial x_{j}}+q^{v}(x) u_{n}^{v} v\right\} d \Omega_{1}=\lambda_{n}^{v} \iint_{\Omega} \Psi^{v} p u_{n}^{v} v d \Omega_{1},
$$

де

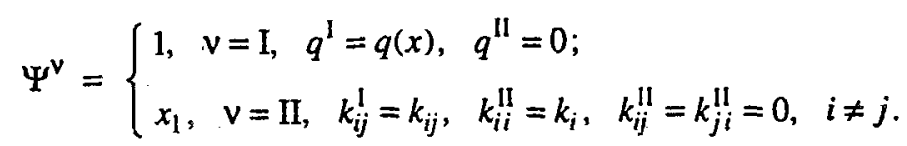

3 (13) випливає, пцо власна функція $u_{n}^{\mathrm{v}}(x)$ в області $\Omega_{1}$ задовольняє диференціальне рівняння

$$
L^{v} u_{n}^{v}(x)=\lambda_{n}^{v} \Psi^{v} \rho u_{n}^{v}(x) \text {. }
$$

Нехаһ̆ тепер $v(x) \equiv 0$ при $x \in \bar{\Omega}_{1}$ i $v(x) \in \stackrel{\circ}{C}^{\infty}\left(\Omega_{2}\right)$. Тоді з (12) випливає, що рівняння (14) справедливе в області $\Omega_{2}$.

Нехай $v(x) є$ довільною функцією на $\partial \Omega_{1} \cap \gamma$. Тоді з урахуванням (14) 3 (12) отримуємо

$$
\int_{\gamma}\left\{\Psi^{v} \sum_{i, j=1}^{2} k_{i j}^{v} \frac{\partial u_{n}^{v}}{\partial x_{j}} \cos \left(n, x_{i}\right)\right\}^{-} v^{-} d \gamma+\int_{\gamma} R \Psi^{\vee}\left[u_{n}^{v}\right]\left(0-v^{-}\right) d \gamma=0 .
$$

Orжe,

$$
\left\{\sum_{i, j=1}^{2} k_{i j}^{v} \frac{\partial u_{n}^{v}}{\partial x_{j}} \cos \left(n, x_{i}\right)\right\}^{-}=R\left[u_{n}^{v}\right], \quad x \in \gamma .
$$

Аналогічно маємо

$$
\left\{\sum_{i, j=1}^{2} k_{i j}^{v} \frac{\partial u_{n}^{v}}{\partial x_{j}} \cos \left(n, x_{i}\right)\right\}^{+}=R\left[u_{n}^{v}\right], \quad x \in \gamma .
$$

Тим самим доведено справедливість твердження.

Наближені узагальнені розв'язки задач I, II на власні значення зручно знаходити за допомогою М СЕ. Для цього доцільно кожну з областей $\bar{\Omega}_{1}, \bar{\Omega}_{2}$ розбити на скінченне число $N_{i}, i=1,2$, трикутників $\bar{e}_{r}^{i}\left(r=\overline{1, N_{i}}, \bar{\Omega}_{i}=\right.$ $\left.=\bigcup_{j=l}^{N_{i}} \bar{e}_{j}^{i}, \bar{e}_{r}^{i} \cap \bar{e}_{l}^{i}=\varnothing, r \neq l ; r, l=\overline{1, N_{i}} ; \bar{\Omega}=\bar{\Omega}_{1} \cup \bar{\Omega}_{2}\right)$. 
Введемо в розгляд простори $H_{k}^{v} \subset H_{\mathrm{v}}$ функціи $v_{k}^{N}(x)$, які неперервні в кожній із областен $\bar{\Omega}_{1}, \bar{\Omega}_{2}$, є повними поліномами степеня $k, k=1,2,3$, змінних $x_{1}, x_{2}$ в кожному з трикутників $\bar{e}_{r}^{i}$ розбиття області $\bar{\Omega}$ і набувають нульових значень в $\Gamma$. Тоді отримаємо алгебраїну узагальнену задачу $v, v=$ $=\mathrm{I}, \mathrm{II}$, на власні значення $[5,7]$

$$
A^{v} \omega^{v k}=\lambda^{v} B^{v} \omega^{v k}
$$

де

$$
\begin{gathered}
A^{v}=A^{v T}>0, \quad B^{v}=B^{v T}>0, \quad A^{v}=\left\{a_{i j}^{v}\right\}_{i, j=1}^{n_{k}}, \quad B^{v}=\left\{b_{i j}^{v}\right\}_{i, j=1}^{n_{k}}, \\
a_{i j}^{v}=\iint_{\Omega}\left\{\Psi^{v} \sum_{l, s=1}^{2} k_{l, s}^{v} \frac{\partial \varphi_{i}^{k}}{\partial x_{s}} \frac{\partial \varphi_{j}^{k}}{\partial x_{l}}+\dot{q}^{v} \varphi_{i}^{k}(x) \varphi_{j}^{k}(x)\right\} d \Omega+\int_{\gamma} R \Psi^{v}\left[\varphi_{i}^{k}\right]\left[\varphi_{j}^{k}\right] d \gamma, \\
b_{i j}^{v}=\iint_{\Omega} \Psi^{v} \rho \varphi_{i}^{k}(x) \cdot \varphi_{j}^{k}(x) d \Omega,
\end{gathered}
$$

$\left\{\varphi_{i}^{k}\right\}_{i=1}^{n_{k}}$ - базнс простору $H_{k}^{N}[4], \omega^{v k}=\left(\omega_{1}^{v k} ; \omega_{2}^{v k}, \ldots, \omega_{n_{k}}^{v k}\right)^{T}$ - невідомі параметри МСЕ (невідомі значення наближеної власної функції $u_{k}^{v N}(x)$ у вузлових точках області $\bar{\Omega}$, чи значення функціi $u_{k}^{v N}(x)$ і відповідних перших $\overline{1}$ частинних похідних - у випадку використання ермітової інтерполяціі). Тут

$$
u_{k}^{v N}(x)=\sum_{i=1}^{n_{k}} \omega_{i}^{v k} \varphi_{i}^{k}(x) \in H_{k}^{N} .
$$

Базисні функції $\varphi_{i}^{k}(x)$, як і функції $v_{k}^{N}(x) \in H_{k}^{N}$, допускають розрнв першого роду на відрізку $\gamma$ розмежування областеи $\Omega_{1}, \Omega_{2}$.

На основі принципу мінімаксу [5] маємо

$$
\begin{array}{cc}
\lambda_{l}^{v}=\min _{S_{l}^{v} \subset H_{v}}\left\{\max _{u \in S_{l}^{v}} R^{v}(u)\right\}, & l=1,2, \ldots, \\
\lambda_{l}^{v N}=\min _{S_{l}^{v N} \subset H_{k}^{N}}\left\{\max _{v_{k}^{N} \in S_{l}^{v N}} R^{v}\left(v_{k}^{N}\right)\right\}, & l=1,2, \ldots, n_{k} .
\end{array}
$$

Отже,

$$
\lambda_{i}^{v} \leq \lambda_{i}^{v N}, \quad i=\overline{1, n_{k}},
$$

де $S_{l}^{v}-l$-вимірний підпростір простору $H_{v} ; S_{l}^{v N}-l$-вимірнии підпростір відповідного простору $H_{k}^{N}, k=1,2,3$.

Для отримання оцінок похнбок наближених власних значень та відповідних наближених власних функцій доцільно ввести в розгляд оператор проектування П лінійних множнн $M^{v}, v=I$, II, на простори $H_{k}^{N}, k=1,2,3$, функцій МСE таким чином:

$$
\alpha^{v}\left(u-\Pi u, v_{k}^{N}\right)=0, \quad u \in M^{v} \quad \forall v_{k}^{N} \in H_{k}^{N} .
$$

Справедлива така лема.

Лема 2. Для кожної функції $u \in M^{v}, v=I$, II, існує $i$ притолуу єдиний елемент $\Pi u \in H_{k}^{N}, k=1,2,3$, такий, чо задовольняе співвідномення (19). Якщо $и(x)$ в кожній з областей $\Omega_{1}, \Omega_{2}$ має обмежені неперервні частинні похідні $(k+1)$-го порядку, то справедлива оцінка 


$$
\|u-\Pi u\|_{W_{3}^{1}} \leq \frac{c h^{k}}{f(\Theta)},
$$

дe $c=$ const $>0, h$ - найбільиа із довжин сторін трикутників $\bar{e}_{r}^{i}$ розбиття області $\bar{\Omega} ; k$-степінь поліномів $M C E ;$ при $k=1 f(\Theta)=\cos \Theta(\Theta-$ половина величини найбільшого з кутів $\left.\bar{e}_{r}^{i}\right) ;$ при $k=2,3 f(\Theta)=\sin \Theta(\dot{\Theta}-$ величина найлсениого з кутів $\bar{e}_{r}^{i}$ ).

Доведення. Для кожної з функцій $u(x) \in M^{v}$ можемо знайти функцію

$$
f^{v}(x)=L^{v} u, \quad u \in M^{v},
$$

і побудувати крайову задачу з розривним розв'язком $u(x)$ на $\gamma$ таким чином:

$$
L^{\nu} u=f^{\nu}(x), \quad x \in \Omega, \quad u \in M^{\nu}, \quad f^{\nu} \in L_{2}(\Omega),
$$

де крайова умова та умови спряження визначаються відповідно співвідношеннями (2)-(4) чи (6)-(8).

Дотримуючись [4], легко бачити, що вибрана функція $u(x) \in M^{\vee}$ при фіксованому $f$ є єдиним розв' язком задачі (21) і єдиною екстремалло функціонала енергіi

$$
F^{v}(v) \doteq \alpha^{v}(v, v)-2\left(f^{v}, v\right), \quad v \in H_{v},
$$

на отриманому раніше енергетичному просторі $H_{\mathrm{v}}$.

Нехай $u(x)$ - розв'язок крайової задачі (21), а $u_{k}^{N}(x) \in H_{k}^{N}-$ її єдиний наближений узагальнений розв'язок. Тоді справедливі рівності

$$
\begin{gathered}
\alpha^{v}\left(u, v_{k}^{N}\right)=\left(f^{v}, v_{k}^{N}\right), \\
\alpha^{v}\left(u_{k}^{N}, v_{k}^{N}\right)=\left(f^{v}, v_{k}^{N}\right) \quad \forall v_{k}^{N} \in H_{k}^{N} .
\end{gathered}
$$

Віднімаючи (24) від (23), одержуємо

$$
\alpha^{v}\left(u-u_{k}^{N}, v_{k}^{N}\right)=0 \quad \forall v_{k}^{N} \in H_{k}^{N} .
$$

Отже, $\Pi u=u_{k}^{N}(x) \in H_{k}^{N}$.

Нехай в $H_{k}^{N}$ існують два елементи $u_{k}^{\mathrm{I} . N} ; u_{k}^{\text {II, } N}$ такі, що

$$
\alpha^{v}\left(u-u_{k}^{\mathrm{I}, N}, v_{k}^{N}\right)=0, \quad \alpha^{v}\left(u-u_{k}^{\mathrm{II} . N}, v_{k}^{N}\right)=0 .
$$

Тоді, вибираючи $v_{k}^{N}=u_{k}^{\mathrm{I}, N}-u_{k}^{\mathrm{II}, N}$, отримуємо суперечність. Отже, існування і єдиність елемента $\Pi u \in H_{k}^{N}$ доведено.

Перейдемо до отримання оцінки (20). Нехай $u$ - розривний класичний розв'язок краюювої задачі (21). Покажемо, що для будь-якої $v(x) \in H_{v}$ справедлива рівність [4]

$$
F^{v}(v)-F^{v}(u)=\|v-u\|_{L^{v}}^{2}, \quad u \in M^{v}, \quad \forall v \in H_{v} .
$$

Дійсно, оскіљкй

$$
\left(f^{v}, v\right)=\left(L^{v} u, v\right)=\langle u, v\rangle^{v}, \quad\left(f^{v}, u\right)=\langle u, u\rangle^{v},
$$

To

$$
\begin{gathered}
F^{v}(v)-F^{v}(u)=\langle v, v\rangle^{v}-2\left(f^{v}, v\right)-\langle u, u\rangle^{v}+2\left(f^{v}, u\right)= \\
=\langle v, v\rangle^{v}-2\langle u, v\rangle^{v}+\langle u, u\rangle^{v}=\|v-u\|_{L^{v}}^{2} .
\end{gathered}
$$


Рівність (26) доведено.

Отже, маємо

$$
F^{v}\left(u_{k}^{N}\right)-F^{v}(u)=\min _{\substack{v_{k}^{N} \in H_{k}^{N} \\ F^{v}}}\left(v_{k}^{N}\right)-F^{v}(u) \leq F^{v}\left(\bar{u}_{k}^{N}\right)-F^{v}(u)=\left\|\bar{u}_{k}^{N}-u\right\|_{L^{v}}^{2},
$$

де $\bar{u}_{k}^{N}(x) \in H_{k}^{N}$ - така функція. що на кожному трикутнику $\bar{e}_{r}^{i}$ триангуляціі області $\bar{\Omega} \epsilon$ повним інтерполяційним поліномом степеня $k$ розв'язку $u(x)$.

Враховуючи оцінки інтерполяції $[8,9]$, умову еліптичності та нерівність Фрідріхса, 3 (27) одержуємо

$$
\left\|u_{k}^{N}-u\right\|_{W_{2}^{1}}^{2} \leq c_{1}\left\|u_{k}^{N}-u\right\|_{L^{\nu}}^{2} \leq c_{1}\left\|\bar{u}_{k}^{N}-u\right\|_{L^{\nu}}^{2} \leq \frac{c^{2} h^{2 k}}{f^{2}(\Theta)} .
$$

Тим самнм встановлено справедливість леми 2.

Припустимо, шо розглядувані задачі не маоть кратних власних значень. Тоді на основі леми 2, слідуючи [5], легко показати страведливість такої теореми.

Теорема. Нехай власні функціi $v_{i}^{v}(x) i=\overline{1, n_{k}}$, задачi v, що допускають розрив на $\gamma$, , нелерервні, неперервно диференційові в кожній з областей $\bar{\Omega}_{1}, \bar{\Omega}_{2}$ і мають неперероні обмежені в $\Omega_{1}, \Omega_{2}$ частинні похідні $(k+1)$-го nорядkу.

Тоді справедливі оцінки

$$
\begin{gathered}
0 \leq \lambda_{l}^{v N}-\lambda_{l}^{v} \leq c h^{2 k} . \\
\left\|v_{l}^{v}-v_{l}^{v N}\right\|_{W_{2}^{1}} \leq c_{1} h^{k}, \quad l=\overline{l, n_{k}},
\end{gathered}
$$

de $k$ - cmeninь поліпомів $M C E$.

Оцінки (28) свідчать про те, що запропоповані обчислювальні схеми для наближеного розв' язку задач па власні заченгя з розривними власними функціями за точністо аснмптотично не гірші за аналогічні, відомі для задач на власні значення з глацкнми власними функціями [5].

Запропоновапі обчнслювальні схеми тестовані на різних задачах на власні значения з розривними власними функціями [7]. Проведені обчислювальні ексгерименти свідчать про те, що зміна параметра $R$ суттєво вплнває на власні гачення розглядуваної задачі з розривними власними функціями. Крім того, в загальному випадку пропорційна зміна параметра $R$ нс викликає пропорційної зміни власінх значень $\lambda_{l}^{\nu}$.

1. Зарубии В. С. Иижеперине метолы решепия залач теплопроводиости. - М. :Эиергоатомнзи(ат, 1983. $-326 \mathrm{c}$.

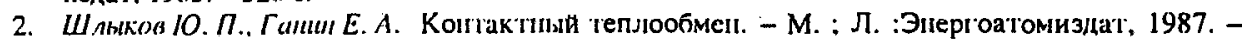
$265 \mathrm{c}$.

3. Коздоба П. А. Метолы решения пелишейних задач теплопрозолиости. - Киев: Наук. лумка. 1975. $-227 \mathrm{c}$.

4. Сереиенко И. В., Скопецкиї В. В., Деїнека В. С. Математическое моделирование и исследо-

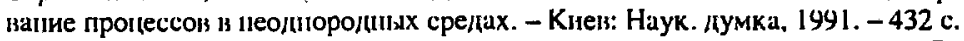

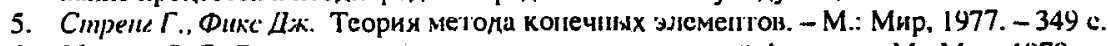

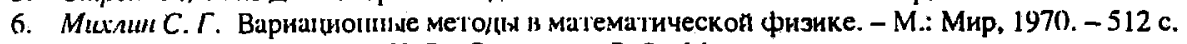

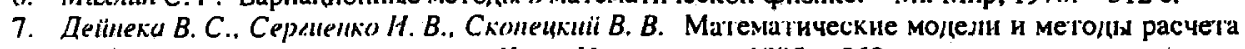
залач с разрынынмии решепи ями. - Киев: Наук. лумка, 1995. - 262 с.

8. Zlaunal $M$. On the finite element method II Numer. Math. - 1968. - 12, № 5. - P. 393-409.

9. Zenisek $A$. Convergence of a finite element procedure for solving boundary value problems of the systems of elliptic equations // Apl. math. - 1969, - 14, № 5, - P. 39-45. пісня /оопралюования - 19.01 .98 